05

\title{
Температура магнитного упорядочения оксида трехвалентного железа $\varepsilon-\mathrm{Fe}_{2} \mathrm{O}_{3}$
}

\author{
(ㄱ Д.А. Балаев ${ }^{1}$, А.А. Дубровский ${ }^{1}$, С.С. Якушкин ${ }^{2}$, Г.А. Бухтиярова ${ }^{2}$, О.Н. Мартьянов ${ }^{2}$ \\ ${ }^{1}$ Институт фризики им. Л.В. Киренского ФИЦ КНЦ СО РАН, \\ Красноярск, Россия \\ ${ }^{2}$ Институт катализа им. Г.К. Борескова СО РАН, \\ Новосибирск, Россия \\ E-mail: dabalaev@iph.krasn.ru
}

(Поступила в Редакцию 11 октября 2018 г.)

Оксид трехвалентного железа $\varepsilon$ - $\mathrm{Fe}_{2} \mathrm{O}_{3}$ является достаточно редкой полиморфной модификацией оксида железа и существует только в виде наночастиц. Этот магнитоупорядоченный материал демонстрирует интересное магнитное поведение: значительную коэрцитивную силу $H_{C}$ при комнатной температуре (до $\sim 20 \mathrm{kOe}$ ), магнитный переход в температурном диапазоне $80-150 \mathrm{~K}$, сопровождающийся резким уменьшением величины $H_{C}$. Ранее считалось, что температура перехода в парамагнитное состояние для $\varepsilon-\mathrm{Fe}_{2} \mathrm{O}_{3}$ составляет около $500 \mathrm{~K}$. Однако недавние исследования показали, что магнитоупорядоченная фаза в $\varepsilon-\mathrm{Fe}_{2} \mathrm{O}_{3}$ существует до более высоких температур, и при этом в окрестности $500 \mathrm{~K}$ происходит ещё один магнитный переход. В натоящей работе на основании данных по намагниченности и температурной эволюции спектров ферромагнитного резонанса показано, что температура перехода в парамагнитное состояние для частиц $\varepsilon-\mathrm{Fe}_{2} \mathrm{O}_{3}$ размерами $3-10 \mathrm{~nm}$ составляет $\sim 850 \mathrm{~K}$.

Работа выполнена при поддержке Российского научного фонда, грант № 17-12-01111.

DOI: 10.21883/FTT.2019.03.47239.277

\section{1. Введение}

Оксид трехвалентного железа $\varepsilon-\mathrm{Fe}_{2} \mathrm{O}_{3}$ является уникальным по своим свойствам магнитным материалом, впервые надежно охарактеризованным в 1998 году [1]. Он существует в виде наночастиц размерами до $\sim 25-40 \mathrm{~nm}[1-11]$, либо нанопроволок [10-19] до $\sim 100 \mathrm{~nm}$, которые демонстрируют значительную коэрцитивную силу (до $\sim 20 \mathrm{kOe}$ и более) при комнатной температуре, а также поглощение электромагнитных волн в миллиметровом диапазоне $[16,10,11]$, что открывает перспективы для практического использования [20-22]. Наночастицы $\varepsilon-\mathrm{Fe}_{2} \mathrm{O}_{3}$ формируются, как правило, в матрице $\mathrm{SiO}_{2}$, при этом используются модификации золь-гель метода $[1,3-5,8,13,23,24]$, метода пропитки силикагеля [25,26]. Кроме того, формирование частиц $\varepsilon-\mathrm{Fe}_{2} \mathrm{O}_{3}$ может происходить в матрице стекла на основе $\mathrm{K}_{2} \mathrm{O}-\mathrm{Al}_{2} \mathrm{O}_{3}-\mathrm{B}_{2} \mathrm{O}_{3}$ [27].

$\varepsilon-\mathrm{Fe}_{2} \mathrm{O}_{3}$ обладает орторомбической нецентросимметричной структурой с пространственной группой Pna $2_{1}$ в области комнатной температуры. Согласно работам [2,9], в диапазоне температур от $\sim 150 \mathrm{~K}$ до $\sim 500 \mathrm{~K}$ магнитную структуру $\varepsilon$ - $\mathrm{Fe}_{2} \mathrm{O}_{3}$ можно считать коллинеарной ферримагнитной (атомы железа находятся в четырех неэквивалентных позициях, связь между подрешетками антиферромагнитная, направлена вдоль оси $c$ [9]). Именно эта магнитная фаза является магнитожесткой. В диапазоне температур $80-150 \mathrm{~K}$ в $\varepsilon$ - $\mathrm{Fe}_{2} \mathrm{O}_{3}$ происходит магнитный переход [2,6,8-11,14,15,17-18,24,28-30], сопровождающийся значительным уменьшением коэрцитивной силы. В области низких (ниже $80 \mathrm{~K}$ ) температур магнитную структуру $\varepsilon$ - $\mathrm{Fe}_{2} \mathrm{O}_{3}$ характеризуют как несоразмерную $[2,9,30]$. Согласно другой точке зрения $[7,8]$, в диапазоне $80-150 \mathrm{~K}$ в $\varepsilon-\mathrm{Fe}_{2} \mathrm{O}_{3}$ происходит метамагнитый переход от одной скошенной антиферромагнитной структуры (слабый ферромагнетизм) к структуре с другим углом скоса.

Долгое время считалось, что переход из парамагнитного в магнитоупорядоченное состояние в $\varepsilon-\mathrm{Fe}_{2} \mathrm{O}_{3}$ происходит в окрестности температуры $\sim 500 \mathrm{~K}$. В работе [30] с помощью магнитных измерений и исследования дифракции нейтронов были получены новые результаты, которые прояснили картину магнитного состояния в оксиде железа $\varepsilon-\mathrm{Fe}_{2} \mathrm{O}_{3}$. По мнению авторов цитированной работы, температура перехода из парамагнитного в магнитоупорядоченное состояние происходит не при $500 \mathrm{~K}$, а при $T_{N 1} \sim 850 \mathrm{~K}$. „Новая“"магнитная фаза, существующая в диапазоне $500-850 \mathrm{~K}$, является магнитомягкой (коэрцитивная сила не превышает $0.5 \mathrm{kOe}$ ) и значения намагниченности меньше таковых для магнитожесткой $(150-500 \mathrm{~K})$ фазы. Описание магнитной фазы в диапазоне $T_{N 2}<T<T_{N 1}$ авторы [30] сводят к тому, что при $T_{N 1} \sim 850 \mathrm{~K}$ происходит ферримагнитное упорядочение двух магнитных железных подрешеток, а при температуpe $T_{N 2} \sim 500 \mathrm{~K}$ происходит ферримагнитное упорядочение двух других железных подрешеток, т. е. ниже $500 \mathrm{~K}$ количество ферримагнитных подрешеток равно четырем.

Отметим, что ранее также регистрировались достаточно высокие температуры магнитного упорядочения в $\varepsilon-\mathrm{Fe}_{2} \mathrm{O}_{3}[3,31,32]$. С одной стороны, такое поведение было интерпретировано как характерная особенность 
частиц $\varepsilon-\mathrm{Fe}_{2} \mathrm{O}_{3}$ экстремально малых размеров [31,32]. $\mathrm{C}$ другой стороны, известно, что $\varepsilon-\mathrm{Fe}_{2} \mathrm{O}_{3}$ редко получается без примесей другого полиморфа - гематита $\left(\varepsilon-\mathrm{Fe}_{2} \mathrm{O}_{3}\right)$, и, возможно, зафиксированная высокая температура магнитного перехода вызвана присутствием гематита $\left(T_{N} \sim 950 \mathrm{~K}\right.$ для объемных образцов). В настоящей работе мы приводим данные, показывающие, что действительно, $\varepsilon$ - $\mathrm{Fe}_{2} \mathrm{O}_{3}$ имеет температуру перехода в парамагнитное состояние, около $850 \mathrm{~K}$, в согласии с выводами работы [30]. Это демонстрируется с помощью двух методик: статических магнитных измерений и ферромагнитного резонанса (ФМР).

\section{2. Получение, характеризация образцов и экспериментальные методики}

Образцы, содержащие наночастицы $\varepsilon-\mathrm{Fe}_{2} \mathrm{O}_{3}$ в матрице силикагеля были получены из сульфатов железа (II) методом пропитки по влагоемкости с последующей сушкой и прокалкой при $900^{\circ} \mathrm{C}$ в воздушной атмосфеpe $[25,33]$. В настоящей работе приводятся данные для образцов, содержащих 0.74 и 3.4 wt.\% железа, которые далее обозначены как 05FS и $3 \mathrm{FS}$ соответственно.

Средний размер частиц $\varepsilon-\mathrm{Fe}_{2} \mathrm{O}_{3}$ по данным изображений просвечивающей электронной микроскопии высокого разрешения (получено помощью микроскопа JEOL JEM-2010 при ускоряющем напряжении $200 \mathrm{kV}$ ) составил 3.4 и $3.8 \mathrm{~nm}$ соответственно. Межплоскостные расстояния, по данным этих изображений, согласуются с параметрами рентгенограмм $\varepsilon-\mathrm{Fe}_{2} \mathrm{O}_{3}$. Гистограммы распределения частиц по размерам для исследованных образцов приведены на вставке рис. 1.

Рентгеноструктурный анализ позволил определить фазу $\varepsilon-\mathrm{Fe}_{2} \mathrm{O}_{3}$ только в образце $3 \mathrm{FS}$ (ввиду малости содержания оксида $\varepsilon-\mathrm{Fe}_{2} \mathrm{O}_{3}$ в образце 05FS). Для идентификации $\varepsilon$-фазы оксида железа была применена мессбауэровская спектроскопия. Результаты анализа мессбауэровских спектров показали, что $\varepsilon-\mathrm{Fe}_{2} \mathrm{O}_{3}$ является единственной железосодержащей фазой в исследованных образцах [34]. Примеси гематита обнаружено не было. Таким образом, исследуемые образцы 05FS и 3FS можно рассматривать как модельные системы наночастиц $\varepsilon-\mathrm{Fe}_{2} \mathrm{O}_{3}$ без посторонних фаз.

Магнитные свойства измерялись с помощью вибрационного магнетометра [35] (температурный диапазон $4.2-300 \mathrm{~K})$ и установки PPMS-6000 (Quantum Design) (температурный диапазон выше $300 \mathrm{~K}$ ). При измерении температурных зависимостей намагниченности $M(T)$ в области температур ниже $300 \mathrm{~K}$ использовались режимы охлаждения без поля (zfc - zero field cooled) и во внешнем поле (fc - field cooled). Экспериментальные данные по намагниченности нормированы на массу оксида железа $\mathrm{Fe}_{2} \mathrm{O}_{3}$.

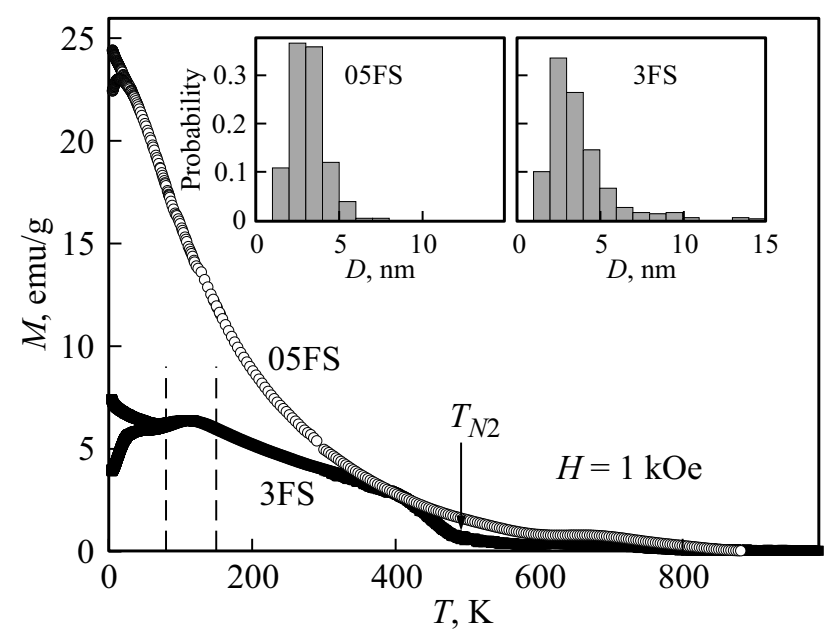

Рис. 1. Температурные зависимости намагниченности $M(T)$ исследованных образцов, содержащих наночастицы $\varepsilon-\mathrm{Fe}_{2} \mathrm{O}_{3}$, в температурном диапазоне $4.2-1000 \mathrm{~K}$. Вертикальные штриховые линии показывают температурный диапазон известного магнитного перехода в $\varepsilon-\mathrm{Fe}_{2} \mathrm{O}_{3}$. На вставках: гистограммы распределения частиц по размерам для исследованных образцов, по данным просвечивающей электронной микроскопии высокого разрешения.

Спектры ФМР были получены на трехсантиметровом спектрометре Bruker ELEXSYS 500, работающем в $X$-диапазоне.

\section{3. Результаты и обсуждение}

\section{1. Намагниченность}

На рис. 1 приведены температурные зависимости намагниченности $M(T)$-образцов в интервале от 4.2 до $1000 \mathrm{~K}$, измеренные во внешнем поле $H=1 \mathrm{kOe}$. Рассмотрим сначала поведение зависимостей $M(T)$ в интервале низких температур, см. рис. 2. Образец 3FS демонстрирует немонотонную зависимость намагниченности от температуры в области от 4.2 до $150 \mathrm{~K}$ (рис. 2). Видимая аномалия в диапазоне $80-150 \mathrm{~K}$ (диапазон помечен вертикальными штриховыми линиями) соответствует известному магнитному переходу в $\varepsilon-\mathrm{Fe}_{2} \mathrm{O}_{3}$. В области температур ниже $80 \mathrm{~K}$ наблюдается сильное влияние термомагнитной предыстории. Образец 05FS показывает другое поведение: монотонный рост намагниченности с уменьшением температуры, наличие четкого максимума зависимости $M(T)$ в условиях zfс при $20 \mathrm{~K}$, и влияние термомагнитной предыстории, начинающееся в области температур в окрестности $20 \mathrm{~K}$. Максимальный размер частиц образца 05FS не превышает $6 \mathrm{~nm}$, и, как показано ранее $[28,32]$, в частицах таких размеров магнитный переход в диапазоне $80-150 \mathrm{~K}$ отсутствует. Наблюдаемый максимум зависимости $M(T)$ zfc соответствует суперпарамагнитной температуре блокировки $T_{B}$ (по нашим данным, значение $T_{B}$ сдвигается в область низких температур при увеличении внешнего поля). 


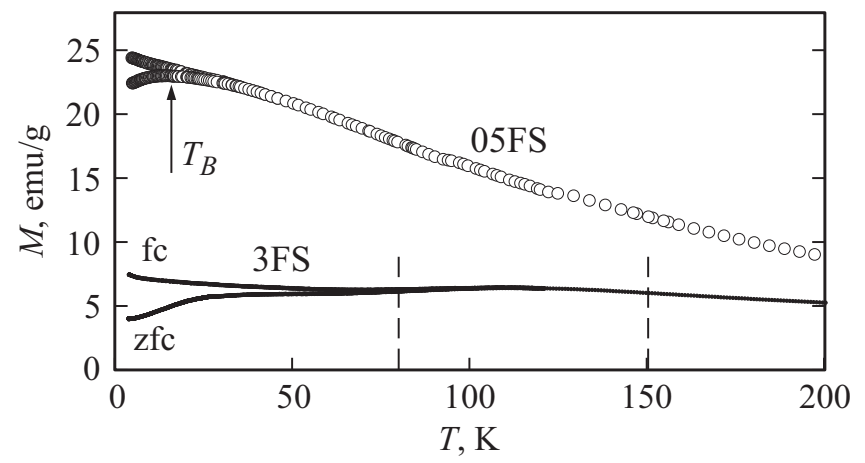

Рис. 2. Температурные зависимости намагниченности $M(T)$ исследованных образцов в области низких температур $(4.2-200 \mathrm{~K})$ при различной термомагнитной предыстории. Вертикальные штриховые линии показывают температурный диапазон известного магнитного перехода в $\varepsilon-\mathrm{Fe}_{2} \mathrm{O}_{3}$. Указана температура суперпарамагнитной блокировки $\left(T_{B}\right)$ для образца $05 \mathrm{FS}$.

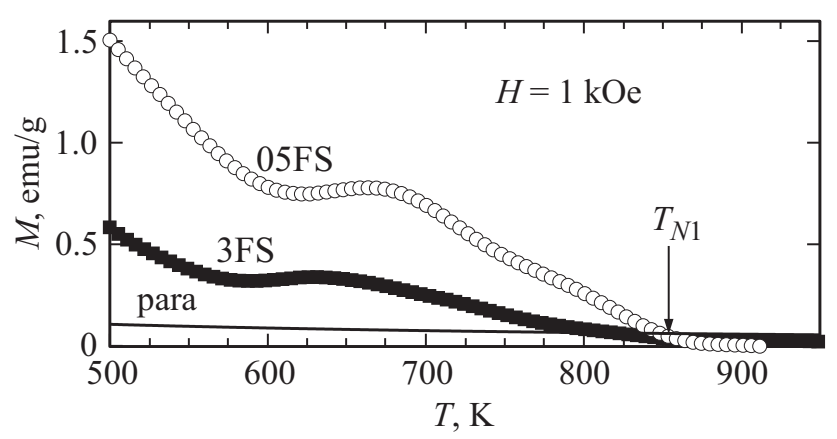

Рис. 3. Температурные зависимости намагниченности $M(T)$ исследованных образцов в области высоких температур $(500-1000 \mathrm{~K})$ - символы. Сплошная линия - ожидаемый вклад от ионов железа (III), находящихся в парамагнитном состоянии. Указана температура перехода в парамагнитное состояние $\left(T_{N 1}\right)$.

Температурная зависимость намагниченности образца 05FS в области температур выше температуры блокировки (рис. 1,2) обусловлена характерным для суперпарамагнитного состояния парапроцессом, при котором зависимость $M(T)$, как правило, пропорциональна $1 / T$. Для образца $3 \mathrm{FS}$, напротив, большая часть частиц остается в заблокированном состоянии вплоть до высоких $(\sim 500 \mathrm{~K})$ температур, что определяет характер зависимости $M(T)$ (типичной для ферримагнетика) - см. рис. 1. Можно видеть резкое уменьшение намагниченности в окрестности температуры $500 \mathrm{~K}$, что помечено на рис. 1 как $T_{N 2}$. Несомненно, что эта особенность соответствует магнитному переходу, который ранее считался переходом в парамагнитное состояние.

Из рис. 1 также видно, что в области высоких (выше $500 \mathrm{~K})$ температур значения намагниченности образцов 05FS и $3 \mathrm{FS}$ - не исчезающе малые величины. На рис. 3 приведены сглаженные зависимости $M(T)$ исследован- ных образцов в области высоких температур. Экспериментальные значения намагниченности существенно превосходят отклик, ожидаемый от атомов железа в $\mathrm{Fe}_{2} \mathrm{O}_{3}$ в парамагнитном состоянии в поле $H=1 \mathrm{kOe}$, рассчитанный по функции Бриллюэна (линия на рис. 3). Это свидетельствует о существовании магнитного порядка в $\varepsilon-\mathrm{Fe}_{2} \mathrm{O}_{3}$ в области высоких температур. Из рис. 3 можно определить, что температура перехода в парамагнитное состояние, обозначенная на рисунке как $T_{N 2}$, составляет около $850 \mathrm{~K}$ для обоих исследованных образцов. Это находится в согласии с данными работы [30].

Как упоминалось выше, для образца 05FS не наблюдается аномалий в окрестности температуры $500 \mathrm{~K}$. Это, наряду с отсутствием магнитного перехода в диапазоне $80-150 \mathrm{~K}$, говорит об измененной магнитной структуре в частицах $\varepsilon-\mathrm{Fe}_{2} \mathrm{O}_{3}$ размером менее $6 \mathrm{~nm}$. Из анализа мессбауэровских спектров этого образца обнаружено существенное отличие распределения катионных позиций от идеальной упорядоченной структуры $\varepsilon-\mathrm{Fe}_{2} \mathrm{O}_{3}[1]$ : заселенность тетраэдрических позиций становится значительно больше [28]. Скорее всего, это вызвано дефектностью структуры частиц $\varepsilon$ - $\mathrm{Fe}_{2} \mathrm{O}_{3}$ таких малых размеров. Тем не менее, из рис. 3 видно, что температура перехода в парамагнитное состояние для образца 05FS также составляет около $850 \mathrm{~K}$.

\section{2. Ферромагнитный резонанс}

Типичные спектры ФМР исследованных образцов, зарегистрированные при различных температурах приведены на рис. 4. В этих спектрах наблюдается узкая интенсивная линия поглощения, соответствующая наличию в образце частиц в суперпарамагнитном состоянии.

Для ансамбля однодоменных магнитных частиц, находящихся в суперпарамагнитном состоянии можно считать, что интегральная интенсивность сигнала поглощения ФМР пропорциональна намагниченности насыщения [36]. На рис. 5 приведены температурные за-
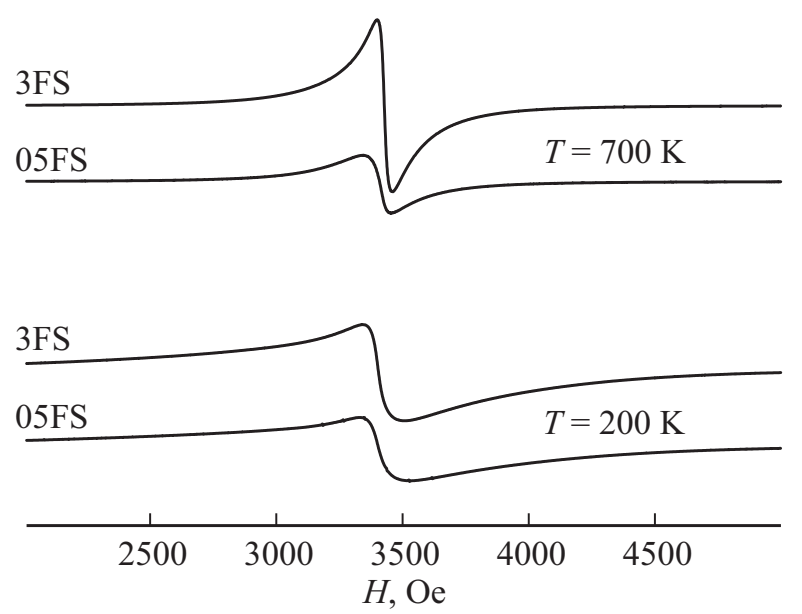

Рис. 4. Спектры ФМР исследованных образцов при указанных температурах. 


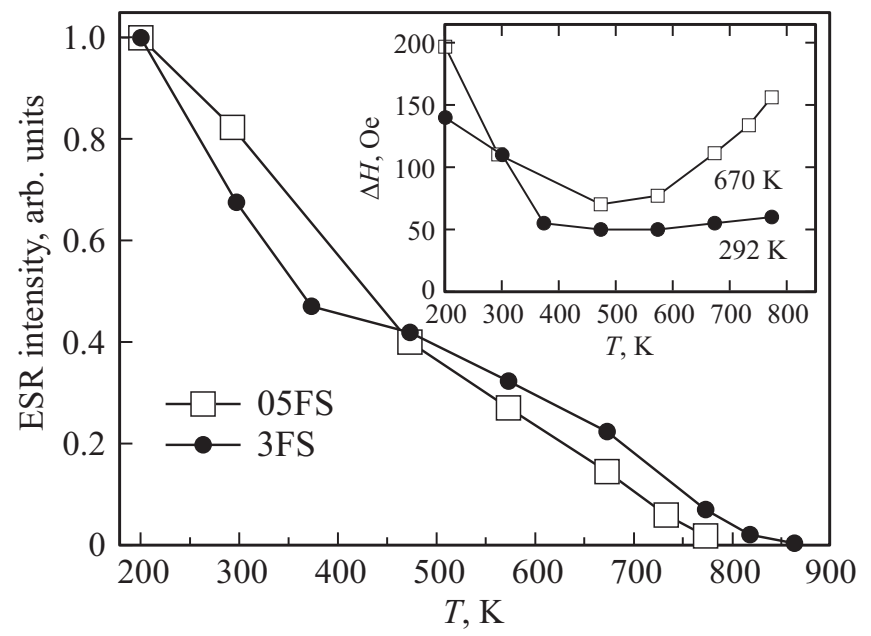

Рис. 5. Температурные зависимости интегральной интенсивности спектров ФМР исследованных образцов в области температур до $900 \mathrm{~K}$. Вставка: ширина линий $\Delta H$ ФМР-спектров как функция температуры.

висимости интегральной интенсивности спектров ФМР для образцов $05 \mathrm{FS}, 3 \mathrm{FS}$. В обоих образцах спектр ФМР наблюдается вплоть до высоких температур, что свидетельствует о наличии магнитного упорядочения в исследуемых образцах в области высоких температур. При этом общий вид зависимости хорошо согласуется с данными магнитных измерений (рис. 3).

На вставке рис. 5 приведены температурные зависимости ширины линии $Ф$ МР $-\Delta H$. В общем случае ширина линии поглощения для индивидуальной однодоменной частицы зависит от кристаллографической анизотропии, формы и условий резонанса. Однако в случае, когда в формировании спектра ФМР участвует ансамбль суперпарамагнитных частиц, большое количество параметров усредняется по причине тепловых флуктуаций [37-39]. Это приводит, в том числе, к усреднению локальных неоднородностей в образце и уменьшению ширины линии при повышении температуры (вставка рис. 5). Однако для исследуемых образцов при температурах выше $500 \mathrm{~K}$ наблюдается качественное изменение характера зависимости $\Delta H(T)$. Это может косвенно свидетельствовать о смене магнитного порядка в $\varepsilon-\mathrm{Fe}_{2} \mathrm{O}_{3}$.

\section{4. Заключение}

Таким образом, измерения намагниченности и температурной эволюции спектров ФМР исследованных образцов, содержащих наночастицы $\varepsilon-\mathrm{Fe}_{2} \mathrm{O}_{3}$ без присутствия других полиморфов оксида железа, позволяют говорить о существовании высокотемпературной магнитоупорядоченной фазы в $\varepsilon-\mathrm{Fe}_{2} \mathrm{O}_{3}$, в согласии с выводами работы [30]. Магнитный порядок сохраняется до температуры $\sim 850 \mathrm{~K}$, причем это наблюдается и для частиц размерами несколько нанометров.

\section{Список литературы}

[1] E. Tronc, C. Chaneac, J.P. Jolivet. J. Solid State Chem. 139, 1, 93 (1998).

[2] M. Gich, A. Roig, C. Frontera, E. Molins, J. Sort, M. Popovici, G. Chouteau, D. Martın Marero, J. Nogues. J. Appl. Phys. 98, 4, 044307 (2005).

[3] M. Popovici, M. Gich, D. Nińanský, A. Roig, C. Savii, L. Casas, E. Molins, K. Zaveta, C. Enache, J. Sort, S. de Brion, G. Chouteau, J. Nogués. Chem. Mater. 16, 5542 (2004).

[4] V.N. Nikolić, M. Tadić, M. Panjan, L. Kopanja, N. Cvjetićanin, V. Spasojević. Ceram. Int. 43, 3147 (2017).

[5] V.N. Nikolic, V. Spasojevic, M. Panjan, L. Kopanja, A. Mrakovic, M. Tadic. Ceram. Int. 43, 7497 (2017).

[6] Д.А. Балаев, С.С. Якушкин, А.А. Дубровский, Г.А. Бухтиярова, К.А. Шайхутдинов, О.Н. Мартьянов. Письма ЖТФ 42, 23 (2016).

[7] E. Tronc, C. Chaneac, J.P. Jolivet, J.M. Greneche, J. Appl. Phys. 98, 053901 (2005).

[8] M. Kurmoo, J.-L. Rehspringer, A. Hutlova, C. D'Orlans, S. Vilminot, C. Estournes, D. Niznansky. Chem. Mater. 17, 1106 (2005).

[9] M. Gich, C. Frontera, A. Roig, E. Taboada, E. Molins, H.R. Rechenberg, J.D. Ardisson, W.A.A. Macedo, C. Ritter, V. Hardy, J. Sort, V. Skumryev, J. Nogués. Chem. Mater. 18, 3889 (2006).

[10] J. Tućek, R. Zboril, A. Namai, S. Ohkoshi. Chem. Mater. 22, 6483 (2010).

[11] L. Machala, J. Tućek, R. Zboril. Chem. Mater. 23, 3255 (2011).

[12] J. Jin, S. Ohkoshi, K. Hashimoto. Adv. Mater. 16, 48 (2004).

[13] S. Ohkoshi, S. Sakurai, J. Jin, K. Hashimoto. J. Appl. Phys. 97, 10K312 (2005).

[14] S. Sakurai, J. Shimoyama, K. Hashimoto, S. Ohkoshi. Chem. Phys. Lett. 458, 333 (2008).

[15] S. Sakurai, A. Namai, K. Hashimoto, S.-I. Ohkoshi, J. Am. Chem. Soc. 131, 18299 (2009).

[16] S. Ohkoshi, S. Kuroki, S. Sakurai, K. Matsumoto, K. Sato, S. Sasaki. Angew. Chem. Int. Ed. 46, 8392 (2007).

[17] А.И. Дмитриев, О.В. Коплак, А. Namai, Н. Tokoro, S. Ohkoshi, Р.Б. Моргунов. ФТТ 56, 1735 (2014).

[18] А.И. Дмитриев, О.В. Коплак, А. Namai, Н. Tokoro, S. Ohkoshi. ФТT 55, 2140 (2013).

[19] А.И. Дмитриев. Письма ЖТФ 44, 17 (2018).

[20] A. Namai, M. Yoshikiyo, K. Yamada, S. Sakurai, T. Goto, T. Yoshida, T. Miyazaki, M. Nakajima, T. Suemoto, H. Tokoro, S. Ohkoshi. Nature Commun. 3, 1035 (2012).

[21] S. Ohkoshi, A. Namai, T. Yamaoka, M. Yoshikiyo, K. Imoto, T. Nasu, S. Anan, Y. Umeta, K. Nakagawa, H. Tokoro. Sci. Rep. 6, 27212 (2016).

[22] S. Ohkoshi, A. Namai, M. Yoshikiyo, K. Imoto, K. Tamazaki, K. Matsuno, O. Inoue, T. Ide, K. Masada, M. Goto, T. Goto, T. Yoshida, T. Miyazaki. Angew. Chem. 128, (2016).

[23] M. Nakaya, R. Nishida, N. Hosoda, A. Muramatsu. Cryst. Res. Technol. 1700110, (2017). DOI: 10.1002/crat.201700110

[24] S.S. Yakushkin, D.A. Balaev, A. A. Dubrovskiy, S.V. Semenov, Yu.V. Knyazev, O.A. Bayukov, V.L. Kirillov, R.D. Ivantsov, I.S. Edelman, O.N. Martyanov. Ceram. Int. 44, 17852 (2018); https://DOI: 10.1016/j.ceramint.2018.06.254.

[25] G.A. Bukhtiyarova, M.A. Shuvaeva, O.A. Bayukov, S.S. Yakushkin, O.N. Martyanov. J. Nanopart. Res. 13, 10, 5527 (2011). 
[26] С.С. Якушкин, Г.А. Бухтиярова, О.Н. Мартьянов. Журн. структур. химии 54, 5, 848 (2013).

[27] O.S. Ivanova, R.D. Ivantsov, I.S. Edelman, E.A. Petrakovskaja, D.A. Velikanov, Y.V. Zubavichus, V.I. Zaikovskii, S.A. Stepanov. J. Magn. Magn. Mater. 401, 880 (2016).

[28] A.A. Dubrovskiy, D.A. Balaev, K.A. Shaykhutdinov, O.A. Bayukov, O.N. Pletnev, S.S. Yakushkin, G.A. Bukhtiyarova, O.N. Martyanov. J. Appl. Phys. 118 (2015).

[29] D.A. Balaev, I.S. Poperechny, A.A. Krasikov, K.A. Shaikhutdinov, A.A. Dubrovskiy, S.I. Popkov, A.D. Balaev, S.S. Yakushkin, G.A. Bukhtiyarova, O.N. Martyanov, Yu.L. Raikher. J. Appl. Phys. 117, 063908 (2015).

[30] J.L. Garcia-Muñoz, A. Romaguera, F. Fauth, J. Nogués, M. Gich. Chem. Mater. 29, 9705 (2017).

[31] S.S. Yakushkin, A.A. Dubrovskiy, D.A. Balaev, K.A. Shaykhutdinov, G.A. Bukhtiyarova, O.N. Martyanov. J. Appl. Phys. 111, 44312 (2012).

[32] D.A. Balaev, A.A. Dubrovskiy, K.A. Shaykhutdinov, O.A. Bayukov, S.S. Yakushkin, G.A. Bukhtiyarova, O.N. Martyanov. J. Appl. Phys. 114, 163911 (2013).

[33] Г.А. Бухтиярова, О.Н. Мартьянов, С.С. Якушкин, М.А. Шуваева, О.А. Баюков. ФТТ 52, 4, 771 (2010).

[34] S.S. Yakushkin, D.A. Balaev, A.A. Dubrovskiy, S.V. Semenov, K.A. Shaikhutdinov, M.A. Kazakova, G.A. Bukhtiyarova, O.N. Martyanov, O.A. Bayukov. J. Supercond. Nov. Magn. 31, 1209 (2017).

[35] А.Д. Балаев, Ю.В. Бояршинов, М.М. Карпенко, Б.П. Хрусталев. ПТЭ 3, 167 (1985).

[36] Г.В. Скроцкий Л.В. Курбатов. Феноменологическая теория ферромагнитного резонанса. В сб.: Ферромагнитный резонанс / Под ред. С.В. Вонсовского. Физ.-мат. лит., М. (1961). C. 25.

[37] R.S. De Biasi, T.C. Devezas. J. Appl. Phys. 49, 2466 (1978).

[38] R. Berger, J. Kliava, J.C. Bissey, V. Baietto. J. Appl. Phys. 87, 7389 (2000).

[39] Yu. L. Raikher, V.I. Stepanov. Phys. Rev. B 50, 6250 (1994).

Редактор К.В. Емщев 